\title{
Factors Affecting Healthcare Access for Older Immigrants: A Qualitative Study with Service Users and Healthcare Social Workers in a Central Canadian City
}

\author{
Hai Luo* and Kimberly Proch \\ Faculty of Social Work, University of Manitoba, USA \\ *Corresponding author: Hai Luo, Assistant Professor, Faculty of Social Work, University of Manitoba, R417B Tier Building, Winnipeg, Manitoba, \\ R3T 2N2, USA
}

Submission: 眥January 18, 2018; Published: 潕 May 10, 2018

\begin{abstract}
Older immigrants are generally found to be less likely than their Canadian-born counterparts to seek hospital care and other healthcare services. This study provides insight into healthcare utilization patterns and barriers from the perspectives of older immigrants and their caregivers, and the healthcare social workers assisting them. The research examines five predisposing factors, nine enabling factors, and physical and mental need factors in Andersen's behavioral model that affect healthcare access. While social workers and multidisciplinary professionals can make efforts to improve mutable factors, such as providing information through community networking and improving the quality and availability of translators in healthcare settings, the factors that acquire mixed levels of mutability-for example, health beliefs and perceived needs, and immigrant status and health insurancerequire long-term commitments of public education and policy change.
\end{abstract}

\section{The Study Context}

According to the 2006 Census, 30\% of seniors in Canada are immigrants [1]. One out of every five adults aged 65 and older in Canada is a first-generation immigrant [1-3]. Visible-minority seniors, many of whom are immigrants, consistently report worse mental and self-rated health than the general older population [4-9]. Regardless, older immigrants are generally less likely than their Canadian-born counterparts to seek healthcare services $[6,7]$. Many studies have been conducted to identify the challenges for vulnerable groups, including older immigrants, in accessing healthcare services [10-12]. Few studies, however, have focused on this group's perceptions of access to healthcare. Various studies present significant findings based on quantitative data generated by surveys or censuses that depict the "results" of a series of social and individual behaviors in utilization of healthcare $[6,10,13]$. Explanations for those behaviors, however, are often missing. This study aims to deepen the understanding of the factors that affect older immigrants' access to health care from the perspective of older immigrants.

\section{Literature review}

Older immigrants have reported several factors-potential barriers to service use-across several studies, including not understanding how to use the healthcare system [14-16]; poor English proficiency as a result of being a new immigrant [2,5,6,11,15-18] ineffective communication with healthcare workers [15,19] lack of social, community, or family supports that may cause isolation and limit access to transportation and assistance with language interpretation $[11,16]$ as well as health, cultural, or personal beliefs $[12,15,20]$. Many studies focused on utilization patterns of older immigrants indicate that beliefs about personal health needs and the appropriate time to ask family and professionals for help can influence when and if services are utilized $[6,7]$. If they believe they should not bother anyone for help-or that they will not be understood well enough to receive adequate careolder immigrants may not access services even when they perceive that help is needed.

Common factors that enable immigrant elders to use services include perceived need or health belief; the greater the perceived need often the more likely healthcare services will be utilized $[11,15,16,20,21]$. Other factors include longer residence in the country and increased English proficiency, family support (interpreter assistance, emotional support, or transportation) [22,17], and knowledge about accessing healthcare services [23]. Access to interpreters and healthcare workers who can effectively communicate with elder immigrants and express understanding also increases service use $[17,13]$. 
Existing research has focused on either revealing low utilization of services [2,19] or analyzing services barriers for this population $[5,18,23]$. Missing from the current research are insights into services and barriers to services from the perspectives of older immigrants, their families, and their caregivers, as well as those of social workers within the healthcare system. These insights will provide a foundation to conceptualize a strategic model for overcoming barriers to older immigrants' access to healthcare services and providing support for their families.

\section{The theoretical framework}

The theoretical framework guiding this study is Andersen's behavioral model of service access [3,24] which has been applied to analyze and predict population service-use patterns since it was developed in the late 1960s $[14,17,21]$. Recently, the model has been adapted to contextualize older immigrants' experience of accessing healthcare and social services [11,20]. The model contains three categories of factor that may impact individuals' use of healthcare services. The predisposing factors include demographic characteristics, social structure (e.g., education, occupation, ethnicity/culture), and health beliefs (e.g., attitudes, values, and knowledge about health) [2]. Enabling factors describe differences in resources for individuals and the community that predict health-service utilization such as availability of health personnel and facilities, and the means and ability(e.g., social support, income, and language competency) to access services $[1,3]$. Needs factors include physical or mental health problems and are often the reasons individuals seeking services $[3,4]$.

The concept of mutability is essential in applying Andersen's behavioral model to understanding older immigrants' access to healthcare $[3,4]$. A factor needs to be changeable to make any efforts for change meaningful. Few factors are static; yet there are different levels of mutability. Demographic factors (i.e., gender, ethnicity) are usually considered low in mutability. Socioeconomic variables are also considered low as occupation, education, and income are not easily changed within a short period of time. Health beliefs are deemed medium mutability, and environmental enabling factors high mutability. Short-term policy and programs targeting mutable factors would effectively improve healthcare access [25]. This study examines the role of culture in Andersen's three categories of factors that influence older immigrants' access to healthcare in a central city in Canada, and how these factors can be manipulated to help promote healthcare access. The results provide insights important to healthcare professionals and policymakers.

\section{Methods}

\section{Data collection and sampling}

Two types of participants from Winnipeg, Manitoba, were recruited to this qualitative study:

A. Older immigrants and their family caregivers, and

B. Social workers in healthcare settings, including hospitals, long-term personal care, homecare, mental healthcare, and other services.
For the first group, purposive sampling was applied to recruit individuals (with their family caregivers) who were 60 years of age or older, were born outside of Canada and immigrated as adults, whose first language was neither English nor French, and who had experience using healthcare services from hospitals, homecare facilities, and community services within the Winnipeg Regional Health Authority (WRHA). Research assistants and the first author contacted and mounted posters at or through WRHA socialwork departments and social-service and healthcare agencies for newcomers from March to October 2015. The research team also visited cultural community centres and religious places, and spread the news when in contact with cultural minority group members such as in taxis.

Research assistants with proficiency in both English and other minority languages (Cantonese, Hindi, Mandarin, and Tagalog), or with cultural minority backgrounds (African, Chinese, Filipino, Indian, Italian, and Trinidadian) were hired to recruit participants and conduct face-to-face interviews. Seventeen older immigrants and 6 of their caregivers/family members were interviewed between February to November 2016. The interviews were guided by two clusters of questions that reflected the two key topics: "What are older immigrants' and their family members' perceptions and experiences of the barriers to access healthcare services?" and "What are older immigrants' and their family members' perceptions of potential strategies to overcome barriers and improve access to health care services?"

All interviews but two were conducted in English. Even when participants were offered the opportunity to be interviewed in their first languages (e.g., Tagalog and Cantonese), older immigrants and their family members preferred speaking English. Altogether, 18 interviews were conducted, with an average length of 61 minutes, ranging from 19 minutes to 105 minutes. To recruit social workers in the healthcare system, a group email was distributed at three different points of time to all social workers in WRHA. The authors recruited social workers who had experiences with and thoughts about barriers immigrant seniors and their families were facing in navigating and using the healthcare system in Winnipeg, and had potential strategies to help overcome those barriers. Social workers self-identified as qualified for the research and contacted the first author for an interview. Fourteen social workers were interviewed either in focus groups or in individual interviews by the first author between September 2015 and March 2016. The interviews were guided by two key questions: "What are social workers' perceptions of the barriers for older immigrants and their families to access healthcare services?" and "What are social workers' perceptions of potential strategies to overcome barriers and improve access to healthcare services for older immigrants and their families?" All interviews were conducted in English. In a total of 24 interviews (focus groups and individual interviews), 18 were conducted with older immigrants and their families, while six were with healthcare social workers. All interviews were audio-taped and transcribed. This study obtained ethical approvals from XXXX Ethics Board and the WRHA Research Ethics Board before the authors initiated recruitment. 


\section{Data analysis}

The transcribed interviews of 23 services users (older immigrants and their families) and 14 service providers were analyzed with the six-step thematic analysis method presented by Braun and Clarke (2006), including familiarization with data, initial coding across the entire data set, collating codes into potential themes, generating a map of themes, defining and refining themes, and write-up of analysis. To analyze the data, the two authors read the transcriptions multiple times and discussed their understanding of the data. They independently analyzed two transcripts of interviews with service users and one with service providers for initial coding and identifying themes then compared their codes and memos. They discovered many similarities of coding in major themes and subthemes and differences in wording to describe the same phenomena. With clarifications, the authors agreed on a code book that they then applied to the work. Throughout the coding and analyzing process, the authors met or emailed to discuss the process and data, and different views of the data were deliberated until agreement was reached.

\section{Result}

Among 23 service users interviewed, 8 were male (34.8\%) and $15(65.2 \%)$ were female. Sixteen were older immigrants who had been direct service users (69.6\%), while 7 (30.4\%) were caregivers and family members of elderly immigrant parents. Older adults' age ranged from 62 to 77, and their years of residence in Canada ranged between 9 and 50. In this group, 5(21.7\%) Chinese, 5 (21.7\%) Filipino, 3 (13.0\%) Indian, 3 (13.0\%) Italian, 2(8.7\%) Spanish, and $3(13.0 \%)$ Trinidadian people were interviewed.

The 14social workers making up the second type of participants had worked in hospitals, rehabilitation centres, homecare services, and community centres for 1 to 33 years (6.5 years on average). The social workers indicated. The social workers reported cases of newer older immigrants (having resided in Canada for less than seven years) and elderly visitors to Canada, in addition to the experiences of older immigrant participants, many of whom were long-term residents in Canada. a clientele of diverse cultural backgrounds, including Central American, Chinese, Egyptian, Filipino, Hungarian, Indian, Israeli, Italian, Peruvian, Portuguese, Russian, and Ukrainian Participants from both groups reported various factors that had affected older immigrants' access to healthcare.

\section{Predisposing factors}

According to the participants in the research, the most influential predisposing factors included personal English proficiency, settlement and immigrant status, personal general cultural beliefs, health beliefs, socioeconomic status, and individual networks. Participants also discussed other predisposing factors, such as demographics.

English proficiency: English proficiency was reported in 20 out of 24 interviews as an important factor affecting older immigrants' access to and use of healthcare. Those who had learned or mastered English before coming to Canada found minimal difficulties in accessing services. The immigrant elders who had worked at paid jobs outside of home generally reported fewer language barriers to access healthcare, even without having learned English before coming to Canada. Older immigrants who had never worked outside of home reported more difficulties in using healthcare, such as not being able to call and make medical appointments or to communicate concerns to healthcare professionals. Participants said that gaining English skills was the first step toward accessing the healthcare system. Inability to communicate prevented service users from effectively navigating the system and taking advantage of services. As a social worker stated, "Some of them have declined a referral because they won't be able to converse at the Adult Day Programs" (Social.Workers_6).

Immigrant status and settlement process: The older immigrants' length of residence in Canada seems to have affected how they use healthcare. Recent immigrants in the early settlement period (0-7 years in Canada) demonstrated more challenges in accessing medical services. While struggling with improving English skills, taking care of families, and/or securing employment to generate stable income, some older immigrants reported becoming ill due to the stress. A rehabilitation social worker noted, "Sometimes people get much stressed and then suddenly they get stroke" (Social.Workers_1). When trying to access health care, newer immigrants, due to limited knowledge of the local system and low English proficiency, usually experienced more stress and anxiety than immigrants who had been here longer. Another issue that affects older immigrants' access to healthcare is immigrant status. All interviewed elders and their family members were permanent residents or citizens while the research was in process, but social worker participants reported several cases in which older adults in critical health conditions were in Canada on visitor visas. Visitors are not covered by Manitoba Health, and sometimes they do not have the proper insurance for unexpected health conditions. Social workers in various settings reported several cases where older visitors and their families were unable to pay healthcare expenses. Though most of medical costs of older immigrants sponsored by families are covered by the healthcare system, there are still fees for some medications and use of ambulance services, if needed-a considerable burden to those living on a tight budget buoyed by family support and limited government subsidy. Social workers tried to help older adults in need of financial assistance by researching different welfare resources, but often the results were disappointing because the programs were only available to citizens and permanent residents. A social worker described the mentality she noticed when helping older adults and their families navigate the healthcare system: People before were more supportive and now people are getting, "Well you know you come to Canada as a visitor, you are supposed to cover whatever, when you are here, you come here, don't expect us to cover for you, you are a visitor. I never go to Mexico or whatever and say come and pay for me." (Social. Workers_1). Immigrant status can be considered a predisposing factor for older immigrants in accessing healthcare as it is an important aspect of socially constructed identity; however, it is heavily related to the policy component in the enabling factors for 
healthcare access. As policy usually takes long periods to change, individuals' characteristics shaped by policy will also remain minimally mutable.

Living arrangements and family challenges: Most older immigrants interviewed were living with their spouses, except for one who lived with his daughter and son-in-law, and one who was a refugee and had always been single. Social worker participants noted that many of their older immigrant clients resided with their children, especially those who were sponsored, or they lived in their own homes but close to their children. Multigenerational living arrangements were reported repeatedly by both service users and social workers to be more pronounced in ethnic cultural communities than in mainstream Winnipeg society. This kind of living arrangement potentially provides solid family support to enable older immigrants' access to the healthcare system. Living in close proximity to family also creates challenges and hardships. For example, one elderly mother was a full-time caregiver to her disabled daughter, while she herself was in needed of medical attention. An older couple lent money to their son residing in the home country, which resulted in severe financial difficulties that prevented them from buying groceries and recommended rehabilitative equipment. Sometimes intergenerational relationships were not reported as positive because the elderly parents and the adult children had to relearn how to live under the same roof in Canada after having had independent lives for years in the home country. The change of roles for the parents-from dependable family heads and breadwinners to dependents (both financially and socially)-caused emotional stress for both parties, which, in turn, led to tense family relationships.

Socioeconomic status: Older immigrants' occupations and financial situations varied. All but two interviewed older immigrants had paid work outside of home. The occupations held by the participants included bookkeeper, engineer, healthcare aide, nurse, teacher, and trades worker. No particular differences among older adults of different professional backgrounds were reported. Five older adults who used to work in the healthcare system, however, indicated more frequently than participants of other occupations that they were aware of healthcare and community resources. This group also commented more frequently on the quality of service they received, and reported that they could advocate for themselves. For example, an elderly woman who previously was a nurse demanded a specialized IV nurse to provide injections for her as she knew her vessels were too difficult to find for unspecialized nurses. None of the service users interviewed mentioned significant financial difficulties in terms of accessing healthcare. Given that most of them were long-term residents in Canada, they received pensions and government benefits as befits the general retired population in Canada, which means they did not need to worry about most medical and medication costs. They might, however, have a hard time paying for additional costs such as ambulance service or certain equipment to enhance quality of life.

Cultural norms and health beliefs: Several older adults from different cultural backgrounds (e.g., Chinese and Italian) were described as selfless parents who put all other family members needs and health concerns before their own, and this trait was notably more pronounced among male family heads than female ones. Some elderly participants stated they would seek religious explanations for illness: "We trust Babaji, the head of the temple, Gurudwara. In the family, I did not know it was rheumatoid. I just expressed my problem to Babaji. Prayed to Babaji" (Indian_1). Usually, this kind of mentality was found among older adults who emigrated from a country where healthcare supply was limited or costly, or among those who had experienced severe financial hardships in their early years. This mindset might delay seeking professional medical intervention. In one case, an elderly gentleman who had never seen a doctor, according to his daughter's recall, had a stroke that might have been avoided if regular checkups had been performed. In some other cases, cultural and religious beliefs may have led to inappropriate professional intervention. For example, in two separate cases with a Filipino family and a Portuguese family, healthcare professionals suggested palliative care instead of aggressive treatment for an elderly parent who was in a critical condition due to a life-limiting illness; family members, however, insisted on full intervention despite prolonged suffering for the parent because the family members believed "it is not up to us to determine what is quality of life....God determines" (Social. Workers_5). Many older immigrants considered their ability to care for themselves as essential to life and said, "I am not going to let everyone else take care of me" (Chinese_1,2,3). Their pride and desire for independence sometimes also delayed consultations with healthcare professionals or minimized their use of health support services, such as home care to facilitate daily activities.

\section{Enabling factors}

Enabling factors can exist at both community and family levels. These factors can both enable and hinder utilization of healthcare services.

Types and quality of healthcare services: Participants reported many different types of healthcare services used by older immigrants, including annual checkups, doctor-on-call, family physicians, hospitals, mental health counselling and psychiatric intervention, rehabilitation for stroke or ophthalmological surgery, walk-in clinics, urgent care, and support programs such as homecare and adult day programs. The quality of services and medical professionals were explicitly acknowledged in 16 out of 24 interviews with service users and social workers. One elderly woman in a wheelchair stressed the convenience of the doctor-oncall in the wintertime, without which she would not have been able to acquire needed medical attention. Many service users indicated that healthcare professionals in both clinic and hospital settings were knowledgeable, trustworthy, patient, kind, caring, prompt in responding to requests, and willing to answer patients' questions and explain medical conditions. An elderly woman appreciated her family doctor's judgment in the diagnosing process of her throat illness. A caregiver described nurses and healthcare aides in a nursing home going the extra mile to learn some words of the language used by her elderly father with Alzheimer's disease, to better communicate with him. Respondents also reported the poor 
quality of specific services, such as that provided by certain family physicians, home care, and personal care services, which resulted in hesitation to continue receiving or obtaining services. Some quality issues were related to healthcare providers' knowledge and judgment, and others related to professional attitude and manners. For example, an older gentleman was brought to an emergency department by his daughter as he had complained about severe back pain; but he was sent home without any tests. A few hours later, they went to see their regular family doctor, who sent him to a hospital for urgent care of heart attack. In another case, an older woman suffering from depression was prescribed ineffective medications by a psychiatrist; her condition worsened until she received medications from a psychiatrist in her home country. Upon returning to Canada, she claimed she did not trust the Canadian psychiatrist enough to accept his prescription, although she continued to visit him on a regular basis. Some participants said their service providers' attitudes and manners pushed them away. For instance, two older immigrants in separate interviews mentioned a family physician, who had the same cultural background as both participants, being constantly rude to and impatient with his patients, and that both eventually stopped seeing the doctor. A caregiver described how her father and a couple of other residents in a personal care home were sometimes untended, sometimes they weren't wearing socks or had food on their faces.

Medical costs and coverage: Many participants expressed their gratitude for Canada's healthcare system, which included all medical intervention and medication costs after a $\$ 500$ deductible, and greatly enabled them to access healthcare when needed. As an older woman said, Our healthcare system is the best system because you don't need to pay to get in. You don't need to consider whether you have money or not to see a doctor. My relatives at home in [the home country], they are the opposite. They must have money to go to a hospital. Only if you have money, you can demand an MRI. Here, I want to get an ultrasound [examination], I can have an ultrasound [examination]. I can get any examinations I want. Because money is not the issue here (Chinese_5). The participants' biggest financial concern was ambulance fees payable by individuals, ranging between $\$ 250$ and $\$ 500$ per use. Some older immigrant participants reported avoiding calling an ambulance even when they had felt they might need it. Dental treatment, eye care, private personal home care, and some alternative healthcare services were not covered by public resources; and none of the participants mentioned having personal insurance for medical costs. Even though these services might increase their quality of life, older adults with limited financial resources reported rejecting them.

Availability of and engagement with healthcare professionals with ethnic cultural backgrounds: Eight interviews indicated that healthcare professionals-family doctors, mental health counsellors, and psychiatrists-who shared their cultures and languages (Cantonese, Hindi, Spanish, and Russian) were available in the community. Working with a professional who spoke their first language eased the older adults' anxiety about going through medical procedures and asking questions about their conditions. However, lack of sufficient healthcare professionals who spoke a language other than the official languages was noted in the interviews as well. One service user described that currently there was only one doctor-in high demand-speaking her ethnic language (Cantonese) available in the community. Several social workers acknowledged that a supply of doctors with multiple language skills was essential to older immigrants' access to healthcare, "not just for recent immigrants, but sometimes there are people who have been here for a number of years...and they are more comfortable to speak to someone in their own language" (Social.Workers_2).

Discrimination in healthcare services: A number of participants stated that, although it was convenient to communicate with doctors speaking the same language, they did not experience any discrimination in accessing the healthcare system. Doctors, nurses, lab technicians, and homecare workers of Caucasian background were reported providing services to older immigrants and treating them as equals. The older adult participants did not believe they had been treated differently from patients of other cultural backgrounds or age groups. Nevertheless, a couple of participants did report discrimination in healthcare and social settings, although neither of them pertained to healthcare professionals. A woman described an incident her husband had encountered in a hospital, where a young Caucasian man cut him off in a waiting line by saying, "I was here first." The husband responded with politeness. "No, my cousins were here first. The Indians [Indigenous people] were here before you" (Filipino_2).

Information and instruction provision: Some participants believed that older immigrants and their families received sufficient information and instructions for accessing healthcare, such as letters reminding them of annual checkups or information about the healthcare system from a settlement agency for newcomers. The majority of the participants (11 out of all 24 interviews; 5 out of 6 interviews with social workers), however, reported a significant gap of information and instruction for older immigrants. Older immigrants and their families said they were not provided with systematic information on how to access healthcare in print materials in English, let alone in their own languages. Some important information, about palliative care, for example, was only available in English materials and was not presented in a manner that was sensitive to the participants' cultural norms. The older immigrant population reported additional barriers in obtaining information as many of them had low computer proficiency, and a significant amount of service information and applications were online. Most important, there was a lack of system guidance and support. As an older man said, "To navigate through the healthcare system you have to be persistent, you have to learn to speak up and ask the appropriate questions, and find the right people to talk to or to guide you." (Trinidadian_3).

Translation services and issues: Participants reported that language barriers were a vital issue in terms of accessing needed healthcare. Two thirds of those interviewed (18 out of 24) explicitly 
indicated language-related difficulties in accessing healthcare. Three older adults and two social worker focus groups reported being aware of the WRHA's Language Access Interpreter Services (LAIS), though utilization of the services was not frequent and consistent among the participants. Two participants believed that some interpreters might not know sufficient medical terminology and mental health vocabulary to accurately explain medical procedures and facilitate communication. Participants also voiced concerns about privacy and confidentiality, especially those from a relatively tight cultural community with a small number of members. In one case, an older man who did not speak English, his daughter, and his hospital care team were discussing a discharge plan for him that involved the possibility of moving into a long-term care facility. Their community recruited an interpreter from LAIS to help facilitate the conversation. When the daughter found out that the interpreter was someone she knew personally, she became "very agitated" because she was uncomfortable about revealing family matters to an acquaintance (Social.Workers_5). The meeting was aborted. A third challenge related to LAIS was mentioned by hospital social workers who said they often received interpreters' assistance via phone, which prevented full communication through non-verbal cues, including body language and facial expressions. Adult children frequently served as interpreters for their elderly parents in the medical setting, including mental health intervention sessions. In most cases, participants said this was convenient, as the children also drove their parents to medical appointments. However, children noted that they usually felt they did not have a sufficient medical vocabulary. In an extreme example, one caregiver participant recalled interpreting for her parents in medical settings when she was 10 or 12 years old, and how scared and stressed she was because of her lack of medical knowledge, despite her fluent English (Italian_1). A critical challenge for children (or other family members) serving as interpreters for older immigrants was the conflict of interest that arose when the older adult and the child did not share the same expectations of care. A hospital social worker noted about one older immigrant's family, They wanted to keep Dad home for the money, that she [the daughter] didn't want to place him, even though he clearly needed to be placed, that was their perspective. He, well you couldn't get an answer out of him. I would try in English many times, the daughter, I mean, getting her to translate, there is a conflict there. (Social.Workers_5).

Transportation: Older immigrants were faced with similar transportation challenges as the general older population. A few programs for seniors, such as day hospital programs and adult day programs, would provide pickup and drop-off services for their attendees, but for the majority of their daily activities, elders needed to make their own transportation arrangements. Most older immigrants did not drive, and they often found taking public transit difficult when their health was declining, especially in winter. To use Handi-Transit provided by public transit for people with disabilities, an older adult had to meet certain criteria. For example, they had to have snow shoveled in front of their houses to be able to access Handi-Transit. It was also reported that HandiTransit services were extremely difficult to navigate due to the fine print on the pass and convoluted instructions for proper use. Older immigrants also needed to overcome another barrier: the Englishlanguage automated phone system. They had to ask their children or a social worker from the community to place a request every time.

Networks in the community: Participants described a variety of networks and supports they could obtain in the community that eventually helped them approach the healthcare system. After moving to Canada, older immigrants met individuals from the same culture in places of worship, ethnic grocery stores, and social activities in the community; they received recommendations for family doctors and information about navigating the system from the networks in their own ethnic communities. Only a few older immigrants indicated they were actively involved in networking with individuals of cultural backgrounds other than their own. Most older immigrants, particularly refugees, reported or were described to be associating only with their own ethnic community, because "it is a little bit easier to trust someone that you have the same culture and the same background with" (Filipino_1).

Family support: Both older immigrant and social worker participants noted that family played the most important role in assisting older immigrants to access healthcare. Spouses and children usually served as the first caregivers to older immigrants, providing transportation to medical facilities, translating conversations with healthcare professionals, and providing daily emotional and practical support. Adult children also took care of elderly parents' finances, arrangements with healthcare agencies, and legal issues, once the parents could no longer do so. Family supports, nevertheless, could also act as hindering factors to healthcare access for older immigrants. Older adults sometimes felt they could rely on their family caregivers instead of taking full advantage of available services. For example, an elderly man receiving homecare services would tell the worker to leave unfinished work to his wife. In other cases, some older adults gave up recovery from a stroke for similar reasons. If our client comes from a belief system that they don't need to get better because their family is going to look after them, it makes it tough to do rehabilitation with them because they are not motivated enough to become independent or to become more independent because they have a system or a family network to fall back on.(Social.Workers_3). Healthcare social workers in various facilities reported similar situations in which cultural beliefs prevented older immigrants and their families from accessing support services such as home care or assisted living. It seemed to social workers that both elderly parents and adult children often refused the idea of engaging external help to care for a family member. In most cases, adult children served as the representatives and interpreters for their older immigrant parents, who had limited English and knowledge about Canada's healthcare system. The children would inform healthcare professionals that they were rejecting home care or a placement in a personal care home, even if they themselves could not maintain a satisfactory level of care for the parents. The work of caring for the elderly usually fell on the shoulders of female family members: 
daughters, daughters-in-law, and wives. When adult children were devoting most of their time and energy to bring a stable income to the family and to young children who might be struggling with adjustment in school and the larger society, the demands of caring for a sick or disabled senior could bring extra stress and work to family members.

\section{Needs factors}

Older immigrant participants reported various health conditions that led to their use of healthcare services. The majority suffered from chronic conditions such as diabetes, chronic heart failure, and pain in the back or chest. Some of them had experienced acute conditions, including heart attack, stroke, and intolerable pain, which brought them to the emergency room. Commonly, those living with chronic diseases experienced health crises that were combined with, or the result of, multiple issues. For example, an elderly woman who had osteoporosis, Parkinson's disease, diabetes, high blood pressure, and high cholesterol was rushed to the emergency room because she had broken her hip (Italian_1). Many participants had been using homecare services, due to their decreased capacity to handle day-to-day activities. Only a few participants indicated they had not yet needed to use intensive healthcare-beyond annual checkups-because of stable health conditions.

It is worth noting that several older immigrants were reported as suffering from mental health problems, including chronic and acute depression, anxiety, psychotic schizophrenia, suicidal ideation, and dementia. In two cases, the mental health interventions of counselling and medications were helpful. However, other service users did not have positive experiences. One elderly woman was prescribed medications for her depression that worsened her mental state until she traveled to her home country and received appropriate medicinal treatment. The interviews with healthcare social workers reflected the findings from service users' interviews that physical and mental health conditions were direct triggers for older immigrants to access healthcare. Their use of healthcare services appeared to result from experiencing acute or chronic conditions that had caused discomfort, and sometimes risks and crises.

\section{Discussion}

Older immigrant healthcare users and healthcare social workers in this study reported a variety of issues in predisposing, enabling, and need factors in accessing healthcare. Five predisposing factors include English proficiency, immigrant status and settlement process, living arrangements and family challenges, socioeconomic status, and cultural norms and health beliefs. Under the enabling category, there are nine factors: types and quality of healthcare services, medical costs and coverage, healthcare professionals of ethnic cultural backgrounds, discrimination, information provision, translation, transportation, networks in the community, and family support. Needs factors were found in both physical and mental health conditions. To better understand these factors, the role of culture and mutability is applied in the following factor analysis.
Consistent with the findings in the literature, English proficiency, immigrant status, length of residence in the host country, and health beliefs were found to be influential for older immigrants to utilize healthcare. Previous findings related to English proficiency were mixed. While some studies indicated low English proficiency was related to low utilization of healthcare [1,2] revealed lower English ability associated with increased visits and suggested that perhaps this was related to the efficiency of the visits when communication between patient and physician is clearer. In this study, low English proficiency was considered a barrier to healthcare. This finding is aligned with the effect of length of residency in the host country; both older immigrants and healthcare social workers believed that the longer an older immigrant had been living in Canada the better he/she could speak English and know the healthcare system, and, in turn, take advantage of it. Longer duration of residency being related to better use of healthcare is generally supported by the literature $[13,23]$.

Immigrant status was considered an important factor in relation to coverage of the public healthcare system. As older adults who had not received permanent resident status would need to pay any medical expenses on their own, whether they or their families would purchase insurance (and the type of health insurance purchased) affected their utilization of healthcare services. Recent immigrants were usually struggling with securing employment for sustainable income, raising children, and improving English while overcoming culture shock. They would delay purchasing insurance or choose minimal coverage for older family members on visitor visas. Policymakers and healthcare social workers may consider, or encourage insurance companies to consider, establishing emergency help programs that facilitate paying for medical costs for visiting older adults, fees that family members could pay back in installments. Immigrant families would also benefit from education programs about local healthcare and insurance systems for older newcomers and their families, so they can make informed decisions.

Health beliefs, influenced by cultural norms and social context, are "individuals' knowledge and values about health care that might influence both their perceived need and actual usage" [1]. Previous studies pointed out the effect of health beliefs in perceived need of service users. For example, many elders expressed the belief that unless their daily lives were being disrupted, no perceived need would exist, and therefore, no service would be accessed [5]. The current study reveals the impact of health beliefs more directly on the motivation to access healthcare than perceived needs. Because accessing healthcare was deemed a time- and money-consuming practice, older immigrants from countries where expensive medical costs were individuals' responsibility would place family members' needs before their own and delay using healthcare. Religious beliefs and related practices could also cause older immigrants to delay seeking professional help; instead, religious intervention and supports were sought. Working with older immigrants and their families, healthcare professionals and social workers need to be aware of cultural beliefs from multiple perspectives. Not only can they affect individuals' perceptions of health conditions, but they 
canal so influence how people evaluate a healthcare system and get a sense of its value.

Among other predisposing factors emerging in the findings of the current study, income was not considered influential in older immigrants' accessing healthcare as most seniors were covered by the healthcare system. Occupation did not seem to be an important factor, either, for the older adults with successful and unsuccessful experiences with healthcare varied in occupational backgrounds, from homemakers to engineers. However, those who had worked or had relatives working in healthcare seemed to be more capable in evaluating the quality of services received and in advocating for themselves or their family members. Health beliefs play a complex role in relation to family support, an enabling factor, as older adults, their children, and healthcare professionals may acquire different opinions and expectations for intervention. Although older immigrants may reject the services considered appropriate by professionals, social workers need to facilitate the treatment process, with respect to service users' self-determination, and maintain a balance between the desires of both service users and providers.

The rejection of certain services, such as palliative care, mental health services, and nursing home placements, may come from immigrant families' cultural or religious norms but could also result from misunderstanding their value. Unfamiliarity with existing services and what services could offer was a common enabling (or hindering) factor in both current and previous studies $[7,13,17,18]$ Providing relevant information for tangible needs, such as doctorson-call and rehabilitation programs, is useful; education about services based on values unfamiliar to immigrant families, however, may require greater community awareness promotion (Authors, in press). For instance, palliative care differs from the conventional idea of aggressive intervention to prolong life in critical conditions; it aims at preserving quality of life from a holistic health perspective. Immigrant families can benefit from learning about the methods and consequences of different types of intervention to make informed choices. Information distribution should not only focus on providing information about and the support to navigate the healthcare system in immigrants' first languages but also address the values and beliefs that support health services and programs.

Availability of translators and interpreters is another frequently mentioned enabling factor [9]. The findings reflect on the advantages and disadvantages of professional translators and family members as translators in healthcare settings. Both

Table 1: Mutability of healthcare behavioral model factors for older immigrants in a Central Canadian City.

\begin{tabular}{|c|c|c|}
\hline Model Category & Model Factor & Degree of Mutability \\
\hline Predisposing & English proficiency & Low \\
\hline & Immigrant Status & Low \\
\hline & Length of Residence & Low \\
\hline & Occupation & Low \\
\hline
\end{tabular}

types of translators can have challenges caused by insufficient medical knowledge and vocabulary. This was particularly true in cases where the older immigrant was from a minority group of a relatively small size, as individuals with both English and minority language proficiency were limited in quantity and quality. Engaging a professional translator from the patient's own community may also trigger problems related to privacy, if the translator knows the service user from community events.

Social network is a vital factor enabling older immigrants' utilization of healthcare. For instance, locating a family doctor is often accomplished through word-of-mouth in minority communities. Older immigrants seem to associate mostly with members of their own ethnic communities, and if there is lack of access information in the community, they may not take full advantage of available healthcare services. Social workers assisting older immigrants and their families in the community and healthcare settings need to encourage them to reach out to support agencies and programs such as Alzheimer's Society, Cancer Care, and Independent Living, which provide support to patients with special needs. Social workers can also help distribute access information about these programs to the community and facilitate navigating the programs, such as giving presentations in community gatherings in churches, temples, or community centers; setting up information booths or tables at popular public events; and providing information at schools to immigrants turn to when in medical need.

The mutability of the above factors varies (Table 1). Immigrant status and duration of residence change overtime, with little control on individuals. Unless government policy on immigration changes, the mutability of these factors is low. English proficiency, occupation, and income can be mutable but depend on a combination of personal, family, and structural factors. For older immigrants, even these factors change but the process may be lengthy. The mutability of health beliefs is mixed. Some beliefs related to certain diseases or conditions can be modified relatively easily-for example, the value of rehabilitation exercises for stroke survivors and the use of homecare workers or medical translators. Other beliefs, especially those related to religious beliefs or cultural norms, would be much less mutable in the short run. For example, it could take a long time and multigenerational efforts to accept or allow different views of death and type of care for a dying person. Health beliefs, as well as English proficiency, immigrant status, and duration of residence, are closely related to individuals' perceived needs. Hence, the mutability of perceived needs is also mixed. children and their parents who are often the first resource older 


\begin{tabular}{|c|c|c|}
\hline & Health Beliefs/Cultural Norms & Mixed \\
\hline Enabling & Quality and Type of Health Care & Medium \\
\hline & Availability of Ethnic Professionals & High \\
\hline & Provision of Information & High \\
\hline & Translation Services & High \\
\hline Need & Transportation Services & Mixed \\
\hline
\end{tabular}

The high quality and type of healthcare greatly enabled older immigrants' use of services, although more medical professionals with ethnic backgrounds and language skills beyond English will likely increase their service use. It is possible that this will happen with the support of a long-term policy of training and recruiting healthcare professionals. Other enabling factors seem relatively more mutable in the short run, compared to predisposing factors, as provision of information, translation, transportation, and increasing community outreach can be carried out through expanding existing programs or establishing new ones (Table 1). For example, access information can be translated in collaboration with service users into minority languages, and video materials can be produced for distribution on community TV channels or individually, free of charge. Social workers at health access centers in different communities can team up with interpreters and reach out to cultural groups to present various medical topics on a regular basis. Social workers, collaborating with other disciplinary professionals, need to advocate for funding to support access to healthcare for older immigrants to promote effective use of healthcare. Enabling factors offer room for improvement in this area.

\section{Conclusion}

This study has examined the predisposing, enabling, and need factors that affect healthcare access for older immigrants. Culture and language are overarching elements that influence across most factors. While social workers and multidisciplinary professionals can make efforts to improve mutable factors (e.g., provision of information through community networking), factors that acquire mixed levels of mutability (e.g., health beliefs and perceived needs) require long-term commitments from various sectors. There are several limitations to this study. First, although the study aimed to include the experiences of older immigrants of as many ethnic groups as possible, some groups (African nationalities, Persian, and Ukrainian) lacked representation in the data collection and findings. These communities seemed reluctant to speak up, or they were not familiar with research and worried about its consequences. Second, the study was unable to recruit recent older immigrants. The reasons could be like those above but also could reflect that recent older immigrants had limited networks outside their ethnic communities and so did not receive the research invitation; or they were uncomfortable about being involved in research due to worries about language barriers. Third, the study did not specifically collect data to differentiate perceived and actual needs of older immigrants.
Nevertheless, the study has provided a cultural understanding of Andersen's model of healthcare access for older immigrants of diverse cultural groups in a central Canadian city, as well as suggestions to social workers working with older adults and their families in healthcare settings and the community. Future research needs to entail older immigrants' access to specific healthcare services-homecare, ambulance, pharmacy, family doctor, urgent care, and hospital services-as the impact of the model components could vary for different types of services.

\section{Acknowledgement}

The studies were supported by the University Research Grants Program (URGP), University of Manitoba; the Endowment Fund of Faculty of Social Work, University of Manitoba; and the Undergraduate Research Grant, University of Manitoba. The studies were approved by University of Manitoba Psychology/ Sociology Research Ethics Board (PSREB) (Protocol \#P2015:066 \& \#P2016:008) and the Winnipeg Regional Health Authority (WRHA) Ethics Committee (Reference No: 2015-045 \& No: 2016-009). The author acknowledges Ms. Vicki Verge and Ms. Meaghan Craven for their valuable support and advice on the study.

\section{References}

1. Ng E, Sanmartin C, Tu J, Manuel D (2014) Use of acute care hospital services by immigrant seniors in Ontario: A linkage study. Health Reports 25(10): 15-22.

2. Durst D (2005) Aging amongst immigrants in Canada: Population drift Canadian Studies in Population 32(2): 257-270.

3. Special Senate Committee on Aging (2007) First interim report: Embracing the challenge of aging. Statistics, Ottawa, Canada.

4. Khan M, Kobayashi K, Lee SM, Vang ZM (2015) Visible minorities in Canadian health data and research: A draft report prepared for presentation at the conference on population change and life course: Taking stock and looking to the future. Ottawa, Canada.

5. Gee EM, Kobayashi KM, Prus SG (2004) Examining the healthy immigrant effect in mid- to later life: Findings from the Canadian Community Health Survey. Can J Aging 23(Suppl 1): S55-S63.

6. Setia MS, Quesnel VA, Abrahamwicz M, Tousignant P, Lynch J (2011) Access to health care in Canadian immigrants: A longitudinal study of the National population health survey. Health Soc Care Community 19(1): 70-79.

7. Hyman I (2007) Immigration and health: Reviewing evidence of the healthy immigrant effect in Canada. Joint Centre of Excellence for Research on Immigration and Settlement (CERIS) Working Paper No. 55, CERIS, Toronto, Canada.

8. Lai DWL (2004a) Depression among elderly Chinese-Canadian immigrants from mainland China. Chin Med J (Engl) 117(5): 677-683. 
9. Lai DWL (2004b) Health status of older Chinese in Canada: Findings from the SF-36 health survey. Can J Public Health 95(3): 193-197.

10. Akresh IR (2009) Health service utilization among immigrants to the United States. Population Research Policy Review 28: 795-815.

11. Aroian KJ, Wu B, Tran TV (2005) Health care and social service use among Chinese immigrant elders. Res Nurs Health 28(2): 95-105.

12. Hadziabdic E, Heikkila K, Albin B, Hjelm K (2009) Migrants' perceptions of using interpreters in health care. International Nursing Review 56(4): 461-469.

13. Nguyen D (2012) The effects of socio-cultural factors on older Asian Americans' access to care. J Gerontol Soc Work 55(1): 55-71.

14. Lai DWL, Surood S (2013) Effects of service barriers on health status of aging South Asian immigrants in Calgary, Canada. Health and Social Work 38(1): 41-50.

15. Koehn S (2009) Negotiating candidacy: Ethnic minority seniors' access to care. Aging \& Society 29(4): 585-608.

16. Stewart M, Shizha E, Makwarimba E, Spitzer D, Khalema EN, et al. (2011) Challenges and barriers to services for immigrant seniors in Canada: You are among others but you feel alone. International Journal of Migration, Health and Social Care 7(1): 16-32.

17. Alizadeh KM, Mathews EM, Hossain SZ (2011) The role of acculturation in health status and utilization of health services among the Iranian elderly in Metropolitan Sydney. J Cross Cult Gerontol 26(4): 397-405.

18. States RA, Susman WM, Riquelme LF, Godwin EM, Greer E (2006) Community health education: Reaching ethnically diverse elders. J Allied Health 35(4): 215-222.

19. Lai DWL, Chau SBY (2007) Predictors of health services barriers for older Chinese immigrants in Canada. Health Soc Work 32(1): 57-65.

20. Kim G, Jang Y, Chiriboga DA, Ma GX, Schonfeld L (2009) Factors associated with mental health service use in Latino and Asian immigrant elders. Aging Ment Health 14(5): 535-542.

21. Andersen RM (1995) Revisiting the behavioral model and access to medical care: Does it matter? J Health Soc Behav 36(1): 1-10.

22. Lai DWL (2004c) Use of home care services by elderly Chinese immigrants. Home Health Care Services Quarterly 23(3): 41-56.

23. Wu Z, Penning MJ, Schimmele CM (2005) Immigrant status and unmet health care needs. Can J Public Health 96(5): 369-373.

24. Andersen RM, Newman JF (1973) Societal and individual determinants of medical care utilization in the United States. Milbank Memorial Fund Quarterly Journal 51(1): 95-124.

25. Braun V, Clarke V (2006) Using thematic analysis in psychology. Qualitative Research in Psychology 3: 77-101.
Creative Commons Attribution 4.0

International License

For possible submissions Click Here
Submit Article

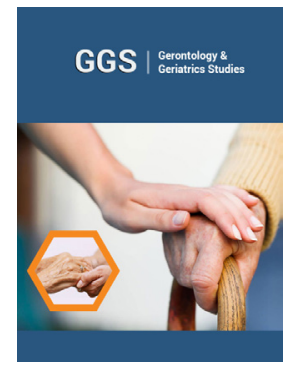

Gerontology \& Geriatrics Studies

\section{Benefits of Publishing with us}

- High-level peer review and editorial services

- Freely accessible online immediately upon publication

- Authors retain the copyright to their work

- Licensing it under a Creative Commons license

- Visibility through different online platforms 